\title{
Multimodal Physical Therapy Approach on Depression, Anxiety, Stress and Quality of Life in Subject with Home Quarantine for COVID-19 Pandemic Attack- A Case Study From India
}

Ramesh Patra ( $\square$ rameshpatra2208@gmail.com )

Lovely Professional University

Biswajit Kanungo

Lovely Professional University

Case Report

Keywords: Anxiety, Breathing Technique, Depression, Physical Therapy, Quality of Life, Stress

Posted Date: May 20th, 2020

DOI: https://doi.org/10.21203/rs.3.rs-28632/v1

License: (c) (1) This work is licensed under a Creative Commons Attribution 4.0 International License. Read Full License 


\section{Abstract}

Purpose: The main purpose of our study was to evaluate the effectiveness of multidisciplinary physiotherapy approach on depression, anxiety, stress and quality of life during lockdown periods for the COVID-19 pandemic attack.

Method: The patient was 56 years female complaining with restlessness and sleep disturbances at night in an outpatient department, Jalandhar Physiotherapy, India. She was a known case of hypertension and grade-2 bilateral knee osteoarthritis. Her level of depression, anxiety and stress was evaluated through DASS-21 (Depression Anxiety Stress Scale) questionnaire. Short Form of health related questionnaire (SF36) was used for the assessment of quality of life. Emotional liberation breathing technique and multimodal physical therapy approaches were used to treat the patient for 2 weeks of periods 3 sessions per week.

Results: After four weeks of follow up the patient was presented with significant improvement in all the parameters depression, anxiety, stress and quality of life (physical functioning, role of limitation-physical health, role of limitation-emotional health, energy, emotional wellbeing, Body pain and general health). These outcomes were reduced the use of analgesics and sedative drugs.

Conclusion: Therefore, the outcomes of this case study concluded that multidimensional therapeutic approach is a beneficial approach to manage depression, anxiety, quality of life, and sleep quality.

\section{Introduction}

According to "World Health Organization" (WHO) the COVID-19 outbreak is a global pandemic [1] which has been started at the end of November in china then gradually spread all over the world. It is one of the lives threating disease affecting the respiratory system [1]. Common symptoms of this disease are dry cough, high fever and difficulty in breathing $[2,3]$. As the medication and vaccine has not been discovered for this disease it is raising concern of the common population about the spread of infection from confirmed COVID-19 positive cases. WHO suggested that social isolation helps to limit the growing number of cases of COVID-19, this has also led to significant fear and anxiety related to spread of infection in the general public. Excessive fear and apprehension of spread of infection can lead to acute stress, anxiety, low quality of life [4]. Though, many organizations are already doing to increase awareness about the societal impact of the on-going pandemic [5]. During this pandemic crisis the possible potential risk factors such as prolonged periods of social isolation, fear of unemployment, economic loss and significant others are increasing due to lockdown $[5,6,7]$.

The relationship between physical activity (PA) and various dimensions of mental health, has received increased attention in recent years [8] and poor mental health is of concern in itself because it may exert a negative effect on individual's quality of life [9].Evidences suggest that there is a direct relationship 
between mental health and sleep quality which is a crucial public health issue in the 21 st century $[10,11]$. Emotional liberation breathing technique (ELB) is a novel therapy which includes both somatic and cognitive elements. In ELB technique is very helpful in increasing the oxygen level in the body which [12]. On the other hand many studies proved that exercise is a gold standard method to manage the subjects with mental health problem. It has been demonstrated that exercise reduces the harmful effects of other stressors when performed at moderate intensities. The pathophysiology of exercise is like neurotransmitter release, neurotropic factor and neurogenesis, and cerebral blood flow alterations are some of the concepts involved [13]. The main purposes of this series was to, the mainly evaluate the effectiveness of multidisciplinary physiotherapy approach on depression, anxiety and quality of life during lockdown periods for the COVID-19 pandemic attack.

\section{Case Description}

\section{Patient information}

The patient was a 56 years old female house wife. She was relatively well before $25^{\text {th }}$ march 2020 then she developed loss of sleep with body heat during day time. She is a known case of hypertension and hyperthyroidism, renal cysts and knee osteoarthritis (Bilateral). There was no history of diabetics. She takes medication for hypertension, thyroid disease and occasionally sedative drugs for sleep. Her past history was when she came back from abroad from her daughter from six month back. After coming from abroad she was developed mild sleep problem after consultation with neurologist the problem was subsided. The problem started again after first week of April month the she consulted with neurologist but this time problem was not subsided even the medication was changed by the neurologist three times in a weeks. Problem was worsening day by day. Then she was assessed by physical therapist and followed the multidimensional physical therapy protocol. The patient was endomorphic on observation. There were no signs of pitting oedema but pain threshold was very less in sub-occipital, and trapezius muscles. She was able to communicate and depicted normal cognitive functioning. She had mild weakness in the lower limb muscles on manual muscle testing and sensory profile was normal.

\section{Outcome measures}

Depression, anxiety, and stress

DASS-21 (Depression Anxiety Stress Scale) was used to assess depression, anxiety and stress. . The participant was approached to utilize a four-point severity/frequency scale to level the degree.

\section{Health Related Quality of Life}

Quality of Life is generally assessed using the SF-36 (MOS 36-item short form health survey) form. Developed by Ware et al., the Turkish validity and reliability adaptation of the form was prepared by Kocyigit et al. The 36 items present on this form reflect eight health-related aspects. 


\section{Intervention}

\section{Emotional liberation breathing}

Emotional Liberation Breathing Technique (ELB) is based on this concept, and an ELB session was divided into 3 big phases to ensure the desired outcome which is a breathing pattern that will be the opposite of all negative breathing pattern s "healing breath" it's like a "general antidote". It is a one hour procedure. The patient was in standing position and the therapist stand just behind the patient. The procedure was started with mouth to mouth breathing means patient was taken deep breathing and exhale through moth until the five sign of ELB were achieved. Then the subject was in rest for 30 minutes. The procedure was performed twice in a week.

\section{Myofascial release Technique}

The patient was asked to lie supine on a couch. The Therapist seated at the head of the table with arms resting on table. First of all neck relaxation was provided by passive movement of the cervical spine. Then therapist places both hands under the neck in such a way that positioned at the base of the skull. The fingertips act as fulcrum for the patient's occiput. The back of skull rested comfortably in the therapist's palm. The patient allows the full weight of head to rest in the palm of therapist's hands. This position is held while physiotherapist waits for the soft tissues to release. The technique was performed twice in a week of alternative day of ELB session.

\section{Exercise Program}

The exercise protocol was carried out under experienced physical therapist. Each exercise session consists of 10-minute warm-up exercises, 40-minute stabilization exercises for cervical spine and scapula, and 10-minute cool-down and stretching exercises, including neck and shoulder girdle muscles. The whole program was carried out 3 days per week for 4 weeks. Then the participants used the latex green color band. Scapulo-thoracic stabilization exercise consists of thoracic bracing, postural alignment and multifidus activation. Dosage and duration for this exercises was 10 repetitions with 8-10 second hold. When the subjects were able to do 15 reputations without any fatigue then they were progress to next color of Thera-Band [16].

\section{Discussion}

In this case study we found that there was significant improvements in all domain of QoL and DASS score. Where improvement shown depression, anxiety and stress respectively 5 points, 8 points, 14 points. In term of depression of In term of health related quality of life- Where improvement shown in physical functioning 45 points, role of limitations due to physical health 75 points, role of limitations due to emotional health 100 points, energy 30 points, emotional well-being 28 points, bodily pain 45 points, general health 30 points. The domain of social life was excluded from the questionnaire due to social distancing. 
Recent evidence supports that ELB is an effective technique to manage physical and mental health. It also claimed that suggests that ELB simultaneously improves a broad range of health markers across multiple physiological systems [14].Another study supports that continuous breathing for about an hour more is going to increase oxygen levels in the blood as breathing increase the level of oxygen inhaled. Oxygen is a natural pain reliever while sometimes increase oxygen contribution to release of tension by reducing the sensitivity of amygdala and cortisol level and increasing serotonin and melatonin level. This process also decreases the dopamine dependency and different types of addictions [15]. Therefore the result of previous study supports the results of the case study. Further extensive research is required to investigate the therapeutic effects of ELB.

\section{Conclusion}

Results of this study have shown that Emotional liberation breathing technique along with Emotional liberation breathing technique and multimodal physical therapy approaches had a positive effect on depression, anxiety, stress and health related quality of life. Therefore, it can be used in clinical practice to manage the patient with depression, anxiety, stress and low quality of life.

\section{Declarations}

\section{Acknowledgements}

Author would like to express heartfelt thanks to all the participants who were participated in this study.

\section{Ethical Clearance and Institutional Review Board Statement}

During the conduct of the case study, human ethical principles as per the World Medical Association's Declaration of Helsinki (2013) and the guidelines of Good Clinical Practice (Indian Council of Medical Research) were observed.

Patient Consent: Participant was informed about the procedure of data collection and written consent form was obtained from all the participant.

\section{Source of Funding-Self funding}

Conflict of Interest -Authors does not have any conflict of interest

\section{References}

1. Cucinotta D, Vanelli M. WHO declares COVID-19 a pandemic. Acta bio-medica: Atenei Parmensis. 2020 Mar 19;91(1):157-60. 
2. Cascella M, Rajnik M, Cuomo A, Dulebohn SC, Di Napoli R. Features, evaluation and treatment coronavirus (COVID-19). InStatpearls [internet] 2020 Mar 8. StatPearls Publishing.

3. Chen H, Guo J, Wang C, Luo F, Yu X, Zhang W, Li J, Zhao D, Xu D, Gong Q, Liao J. Clinical characteristics and intrauterine vertical transmission potential of COVID-19 infection in nine pregnant women: a retrospective review of medical records. The Lancet. 2020 Mar 7;395(10226):809-15.

4. Wang C, Pan R, Wan X, Tan Y, Xu L, Ho CS, Ho RC. Immediate psychological responses and associated factors during the initial stage of the 2019 coronavirus disease (COVID-19) epidemic among the general population in China. International journal of environmental research and public health. 2020 Jan;17(5):1729.

5. Sahoo S, Bharadwaj S, Parveen S, Singh AP, Tandup C, Mehra A, Chakrabarti S, Grover S. Self-harm and COVID-19 Pandemic: An emerging concern-A report of 2 cases from India. Asian Journal of Psychiatry. 2020 Apr 16.

6. Goyal K, Chauhan P, Chhikara K, Gupta P, Singh MP. Fear of COVID 2019: First suicidal case in India!. Asian journal of psychiatry. 2020 Mar;49:101989.

7. LAW K. COLLEGE Approved by Bar Council of India and affiliated to Karnataka State Law University, Hubli. KLE Society.

8. Biddle SJ, Ciaccioni S, Thomas G, Vergeer I. Physical activity and mental health in children and adolescents: An updated review of reviews and an analysis of causality. Psychology of Sport and Exercise. 2019 May 1;42:146-55.

9. Fox KR. The influence of physical activity on mental well-being. Public health nutrition. 1999 Mar;2(3a):411-8.

10. Baglioni C, Battagliese G, Feige B, Spiegelhalder K, Nissen C, Voderholzer U, Lombardo C, Riemann D. Insomnia as a predictor of depression: a meta-analytic evaluation of longitudinal epidemiological studies. Journal of affective disorders. 2011 Dec 1;135(1-3):10-9.

11. Spira AP, Covinsky K, Rebok GW, Punjabi NM, Stone KL, Hillier TA, Ensrud KE, Yaffe K. Poor sleep quality and functional decline in older women. Journal of the American Geriatrics Society. 2012 Jun;60(6):1092-8.

12. Bach D, Groesbeck G, Stapleton P, Sims R, Blickheuser K, Church D. Clinical EFT (Emotional Freedom Techniques) improves multiple physiological markers of health. Journal of evidence-based integrative medicine. 2019 Feb 14;24:2515690X18823691.

13. Mikkelsen K, Stojanovska L, Polenakovic M, Bosevski M, Apostolopoulos V. Exercise and mental health. Maturitas. 2017 Dec 1;106:48-56.

14. Bach D, Groesbeck G, Stapleton P, Sims R, Blickheuser K, Church D. Clinical EFT (Emotional Freedom Techniques) improves multiple physiological markers of health. Journal of evidence-based integrative medicine. 2019 Feb 14;24:2515690X18823691.

15. Lenique F, Habis M, Lofaso F, Dubois-Rande JL, Harf A, Brochard L. Ventilatory and hemodynamic effects of continuous positive airway pressure in left heart failure. American journal of respiratory and critical care medicine. 1997 Feb;155(2):500-5. 
16. Celenay ST, Akbayrak T, Kaya DO. A comparison of the effects of stabilization exercises plus manual therapy to those of stabilization exercises alone in patients with nonspecific mechanical neck pain: a randomized clinical trial. journal of orthopaedic \& sports physical therapy. 2016 Feb;46(2):44-55.

\section{Tables}

\begin{tabular}{|l|l|}
\hline \multicolumn{2}{|l|}{ Table 1: Demographic characteristics of all patients } \\
\hline Age $(\mathrm{y})$ & 56 \\
\hline Weight $(\mathrm{kg})$ & 66 \\
\hline Height $(\mathrm{cm})$ & 163 \\
\hline Gender & Female \\
\hline Body mass Index $(\mathrm{kg} / \mathrm{m} 2)$ & 24.11 \\
\hline Smoking history & No \\
\hline Marital status & Married \\
\hline Occupational history & House Wife \\
\hline Education history & Graduation \\
\hline Socio-economical history & Well Established \\
\hline
\end{tabular}


Table 2 comparison of outcome measures for before and after the treatment

\begin{tabular}{|c|c|c|c|c|}
\hline \multicolumn{2}{|c|}{ Outcome Measures } & Before the treatment & After the treatment & Difference \\
\hline \multicolumn{2}{|c|}{ Depression } & 10 & 5 & 5 \\
\hline \multirow{2}{*}{ Anxiety } & 11 & 3 & 14 \\
\hline \multirow{2}{*}{ Stress } & PF & 18 & 4 & 45 \\
\cline { 2 - 6 } & RL-PH & 45 & 90 & 75 \\
\cline { 2 - 5 } & RL-EH & 25 & 100 & 100 \\
\cline { 2 - 6 } & ENG & 00 & 100 & 55 \\
\cline { 2 - 6 } & EWB & 30 & 85 & 28 \\
\cline { 2 - 6 } & BP & 40 & 68 & 45 \\
\cline { 2 - 6 } & GH & 45 & 90 & 30 \\
\hline
\end{tabular}

PF: physical functioning, RL-PH: role of limitation-physical health, RL-EH: role of limitation-emotional health,

EN: energy, EWB: emotional wellbeing, SF: social life, BP: Body pain, GH: general health 